PAPER

\title{
The spasticity paradox: movement disorder or disorder of resting limbs?
}

\section{J A Burne, V L Carleton, N J O'Dwyer}

J Neurol Neurosurg Psychiatry 2005;76:47-54. doi: 10.1136/jnnp.2003.034785

See end of article for authors' affiliations

.....................

Correspondence to: $\operatorname{Dr} J$ A Burne, University of Sydney, PO Box 170, Lidcombe, NSW 1825 Australia; J.Burne@fhs. usyd.edu.au

Received

19 December 2003

In revised form

22 April 2004

Accepted 28 April 2004
Background: Spasticity is defined/assessed in resting limbs, where increased stretch reflex activity and mechanical joint resistance are evident. Treatment with antispastic agents assumes that these features contribute to the movement disorder, although it is unclear whether they persist during voluntary contraction.

Objectives: To compare reflex amplitude and joint resistance in spastic and normal limbs over an equivalent range of background contraction.

Methods: Thirteen normal and eight hemiparetic subjects with mild/moderate spasticity and without significant contracture were studied. Reflex and passive joint resistance were compared at rest and during six small increments of biceps voluntary contraction, up to $15 \%$ of normal maximum. A novel approach was used to match contraction levels between groups.

Results: Reflex amplitude and joint mechanical resistance were linearly related to contraction in both groups. The slopes of these relations were not above normal in the spastic subjects on linear regression. Thus, reflex amplitude and joint resistance were not different between groups over a comparable range of contraction levels. Spastic subjects exhibited a smaller range of reflex modulation than normals because of decreased maximal contraction levels (weakness) and significant increases of resting contraction levels. Conclusions: Spasticity was most evident at rest because subjects could not reduce background contraction to normal. When background contractions were matched to normal levels, no evidence of exaggerated reflex activity or mechanical resistance was found. Instead, reduced capacity to modulate reflex activity dynamically over the normal range may contribute to the movement disorder. This finding does not support the routine use of antispastic agents to treat the movement disorder.
T he paradox in relation to spasticity is that it has been clinically defined ${ }^{1}$ and primarily studied in resting limbs, yet its clinical management is directed mainly at the associated movement disorder. Current directions in the treatment of spasticity include the use of antispastic agents such as botulinum toxin and baclofen to reduce overactive muscle activity. The rationale for this approach is that sustained overactivity in some way limits limb performance. This view is supported by the clinical definition of spasticity and numerous studies that report increased mechanical resistance and augmented tonic stretch reflexes (TSR) during passive joint rotation, particularly after stroke $\mathrm{e}^{2-7}$ and cerebral palsy. ${ }^{89}$ On this basis, spastic limbs can be clearly distinguished from normal limbs at rest, in which slow stretches generally fail to elicit signs of increased tone or significant levels of TSR activity. ${ }^{40-14}$

Although it is more relevant to treatment, relatively little is known of spasticity in contracting muscle. Even a small voluntary background contraction leads to prominent TSR activity and increased passive resistance in normal limbs, ${ }^{7815} 16$ and there is then no clear demonstration that reflex magnitude $e^{2417-19}$ and joint resistance ${ }^{20}$ are significantly higher in spastic limbs. Hence, it is unclear how, or if, spasticity contributes to the movement disorder in the affected limbs. It is possible, without clear evidence to the contrary, that the defining features of spasticity are a phenomenon confined to resting limbs. A more detailed knowledge of the properties of spastic muscle during active contraction is needed to guide future directions in treatment.

This paper reports a systematic study of the effect of muscle contraction on spasticity and outlines the implications for treatment. More specifically, we tested the possibility that a small rise in resting contraction levels could contribute to the observed increase in TSR activity and passive resistance in spastic muscles. In normal subjects, reflex amplitude and joint stiffness are linearly related to contraction level, at least for contractions below 50\% of maximum. ${ }^{16}{ }^{21-29}$ Therefore, it was important to assess the effect of background contraction on reflex amplitude and joint resistance in normal and spastic limbs over a comparable range of contraction levels.

In practice, several problems complicate attempts to match contraction levels in normal and spastic limbs. Spastic limbs typically exhibit a much reduced range of contraction and subjects find it difficult to hold a target level of voluntary contraction or torque. In addition, traditional methods of electromyogram (EMG) normalisation are invalidated in weak spastic subjects, who will use a larger proportion of their maximum voluntary contraction (MVC) to achieve a given torque output. The problems associated with matching contraction levels in normal and spastic limbs have received little attention in past studies of reflexes and joint stiffness, and the effect of very small background contractions on reflex amplitude and joint resistance in spastic or normal subjects is not known.

Our study used a novel approach to the problem of controlling for differences in contraction level between subjects. We also used analysis techniques that permitted simultaneous estimation of reflex amplitude and resistive torque, so that these two variables could be directly correlated in each subject. TSR amplitude and mechanical resistance at the elbow were estimated in spastic and normal limbs at rest and at six small incremental levels of background isometric

Abbreviations: EMG, electromyogram; TSR, tonic stretch reflex; MVC, maximum voluntary contraction 
biceps muscle contraction to a maximum of $15 \%$ of MVC. These data permitted regression analysis of the relation between reflex amplitude and contraction level, and also between mechanical joint resistance and contraction level over this range in individual subjects. The derived slopes of these relations provide the first comparison of reflex amplitude and resistive torque in spastic and normal limbs over a similar range of contraction levels. Extrapolation of the regression lines back to zero contraction level gave estimates of reflex amplitude and mechanical joint resistance at a theoretical zero contraction level. Changes in passive tissue properties secondary to muscle shortening were excluded from consideration because the study was based on limbs that were free of significant contracture.

Previous studies have used a wide range of perturbations in terms of amplitude and frequency content, and this must have contributed to the conflicting results obtained. Reflex amplitude and joint stiffness show non-linear power relations with both stretch amplitude and frequency. ${ }^{20} 21233031$ Recent studies have used peak to peak stretch amplitudes below $5^{\circ}$ and frequencies above $30 \mathrm{~Hz}$. Such stretches therefore reflect the highly non-linear properties of the stretch reflex, leading to reflex amplitude and joint stiffness estimates that are larger by an order of magnitude than those encountered in the clinical testing of joint resistance. In our current study, the stretch parameters were selected to reflect as much as possible the range used in clinical testing, so that the results would have relevance to spasticity as clinically defined.

\section{MATERIALS AND METHODS \\ Subjects}

Thirteen normal subjects (mean age, 45.8; SD, 9.3 years) and eight spastic hemiparetic subjects (mean age, 57.6; SD, 7.9 years) participated in our study. Table 1 summarises their demographic information. The inclusion criteria for normal subjects were that they fell within the approved age range (20-70 years) and were free of neurological and musculoskeletal abnormalities. Inclusion criteria for spastic hemiparetic subjects were that they were greater than one month post-stroke, possessed clinical signs of spasticity, accompanied by a minimum Ashworth score of 1, had sufficient strength and range of motion at the elbow joint to perform the experimental task, and could follow the test instructions. Subjects with major sensory impairment, antispastic or botulinum toxin medications, or an inability to attain the correct testing position as a result of soft tissue contracture and/or discomfort were excluded.

Anthropometric data, including segmental arm lengths and upper, mid, and lower forearm circumferences were collected for each subject so that calculations of elbow joint resistance could be normalised for the effects of limb volume. Our study was approved by the human ethics committee of the University of Sydney and informed consent was obtained according to the Declaration of Helsinki.

\section{Stretch perturbation}

Subjects were seated in a semi-reclining chair, with their right arm (normal subjects) or affected arm (stroke subjects) in $90^{\circ}$ of shoulder abduction and $90^{\circ}$ of elbow flexion. The height of the chair was adjusted so that the correct arm position could be attained. The semi-pronated forearm and hand were supported in a horizontally orientated adjustable cast, which was connected to a computer controlled DC torque-servo motor (Baldor Instruments, Sydney, Australia) via a vertically aligned motor shaft. The axis of rotation of the elbow joint was aligned with the axis of rotation of the motor shaft. Rotation about the elbow joint was constrained to the horizontal plane to eliminate gravitational effects.

Because spasticity can be detected by manual clinical testing and is clinically defined by this method, ${ }^{1}$ we used a range of stretch amplitudes and frequencies comparable to those used clinically, achieving a maximum velocity of $90 \%$ s. Otherwise, because reflex amplitude and resistive torque vary in a non-linear manner with stretch frequency and amplitude, estimates may be obtained that are larger by an order of magnitude than the values expected from clinical testing or reported here.

A broadband signal with an irregular profile and a frequency content of $0.1-3.0 \mathrm{~Hz}$ drove the perturbation. The signal was digitally generated from a random number sequence, which was subsequently low pass filtered by a fourth order Butterworth filter with a $3.0 \mathrm{~Hz}$ cutoff frequency. The resulting perturbation of the elbow was centred on $90^{\circ}$ of elbow flexion and had a maximal amplitude $\pm 15^{\circ}$. Preliminary studies showed that normal subjects could not voluntarily track the irregular perturbation profile on request. In addition, the results to be reported here showed the reflex EMG response to be consistently phase advanced with respect to the perturbation, confirming that voluntary muscle activity did not contribute significantly to the measures of reflex amplitude derived from the correlation analysis.

\section{Recordings}

Joint position was measured by a precision potentiometer (AXEM MC19S) attached to the lower end of the motor shaft. Resistive torque was measured by monitoring the amount of current supplied to the motor, because torque output is directly proportional to the current supplied. Before the experiment, the torque motor signal was calibrated by suspending a range of known weights from the manipulandum,

Table 1 Demographic information

\begin{tabular}{|c|c|c|c|c|c|c|c|c|c|c|c|}
\hline Subjects & $\begin{array}{l}\text { Age } \\
\text { (years) }\end{array}$ & Sex & $\begin{array}{l}\text { Location } \\
\text { of CVA }\end{array}$ & $\begin{array}{l}\text { Length of } \\
\text { treatment* } \\
\text { (months) }\end{array}$ & $\begin{array}{l}\text { Time after } \\
\text { stroke } \\
\text { (months) }\end{array}$ & $\begin{array}{l}\text { Handedness } \\
\text { (L/R) }\end{array}$ & $\begin{array}{l}\text { Arm } \\
\text { tested } \\
\text { (L/R) }\end{array}$ & $\begin{array}{l}\text { Ashworth } \\
\text { scale } \\
(0-4)\end{array}$ & Contracture & $\begin{array}{l}\text { MAS advanced } \\
\text { hand activities }\end{array}$ & $\begin{array}{l}\text { Antispastic } \\
\text { medication }\end{array}$ \\
\hline \multicolumn{12}{|l|}{ Stroke } \\
\hline 14 & 46 & $\mathrm{~F}$ & (L) MCA & 5 & 22 & L & $\mathrm{R}$ & 1.5 & Absent & 1 & Nil \\
\hline 15 & 48 & $\mathrm{~F}$ & (R) MCA & 4.5 & 60 & $\mathrm{R}$ & $\mathrm{L}$ & 2 & Absent & 0 & Nil \\
\hline 16 & 57 & $M$ & (L) $M C A$ & 4 & 86 & $\mathrm{R}$ & $\mathrm{R}$ & 3 & Present & 3 & Nil \\
\hline 17 & 58 & $M$ & (L) MCA & 1.5 & 26 & $\mathrm{R}$ & $\mathrm{R}$ & 1 & Absent & 5 & Nil \\
\hline 18 & 58 & $M$ & (L) MCA & 12 & 60 & $\mathrm{R}$ & $\mathrm{R}$ & 2 & Absent & 3 & Nil \\
\hline 19 & 59 & $\mathrm{~F}$ & (L) MCA & 3.5 & 60 & $\mathrm{R}$ & $\mathrm{R}$ & 1 & Absent & 0 & Nil \\
\hline 20 & 70 & $M$ & (R) MCA & 2 & 7 & $\mathrm{R}$ & $\mathrm{L}$ & 1.5 & Absent & 4 & Nil \\
\hline 21 & 65 & $M$ & (L) MCA & 2.5 & 16 & $\mathrm{R}$ & $\mathrm{R}$ & 1.5 & Absent & 6 & Nil \\
\hline \multicolumn{12}{|l|}{ Normal } \\
\hline $1-13$ & $21-58$ & $6 \mathrm{~F}, 7 \mathrm{M}$ & - & - & - & $12 \mathrm{R}, 1 \mathrm{~L}$ & $\mathrm{R}$ & 0 & - & - & - \\
\hline
\end{tabular}

*Physiotherapy or occupational therapy.

CVA, cerebrovascular accident; L, left; MAS, Motor Assessment Scale; MCA, middle cerebral artery; R, right. 


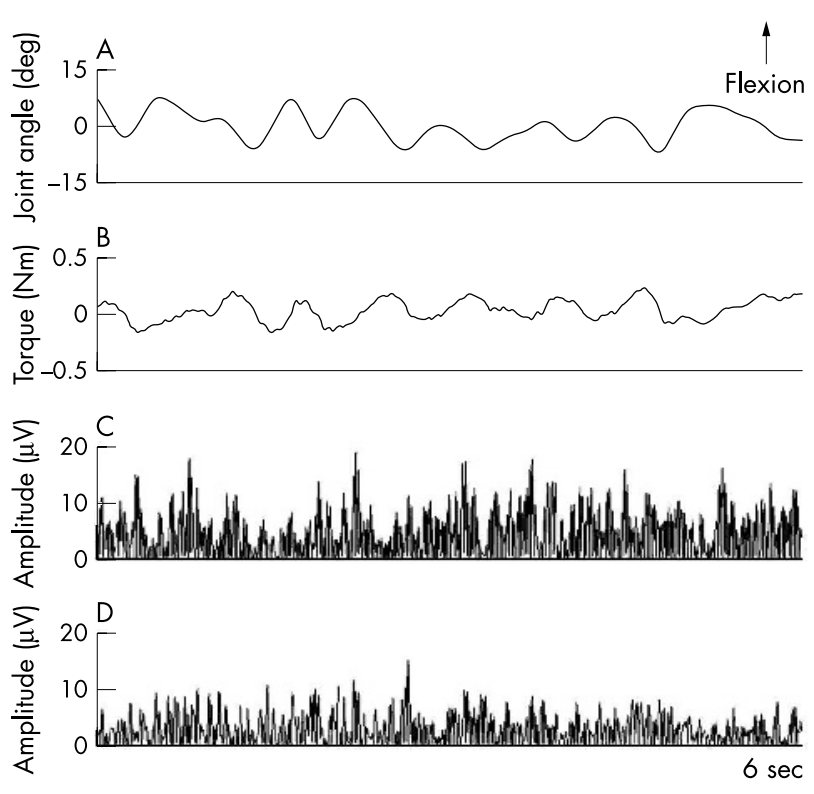

Figure 1 Samples of (A) joint angle, (B) resistive torque, (C) high pass filtered and rectified biceps electromyogram (EMG), and (D) rectified and filtered triceps EMG recorded during perturbation of the elbow by a broadband stimulus $(0-3 \mathrm{~Hz})$ in a normal subject during a $2.5 \%$ maximum voluntary contraction of biceps. Flexion is represented by an upward deflection of the position trace.

and was found to have a linear relation to torque over the testing range. Surface EMG was measured by bipolar silversilver chloride electrodes, placed $2 \mathrm{~cm}$ apart over the central belly of the biceps brachii and the lateral head of the triceps muscles. Elbow joint angle, resistive torque (analogue filtered, $0.1-400 \mathrm{~Hz}$ band pass) and flexor and extensor surface EMG (analogue filtered, 5-400 Hz band pass) were recorded and digitised at $1000 \mathrm{~Hz}$ (Amlab2, Sydney, Australia).

\section{EMG calibration}

A series of MVCs of elbow flexor and extensor muscles was recorded before testing and mean MVCs were calculated digitally by software. Because MVC levels were not comparable in normal and stroke subjects, as a result of weakness in the stroke group, a second calibration method was preferred. Subjects were required to oppose several cycles of a $2 \mathrm{Nm}$ peak to peak sinusoidal variation in torque at $0.3 \mathrm{~Hz}$, applied about the elbow joint by the torque-servo motor. Concurrent measures of rectified flexor and extensor EMG activity were correlated against the torque signal. The gain of the EMG/ torque relation was thus determined and used subsequently to convert the EMG signals from each subject into equivalent torque units. This was later used to normalise the flexor and extensor EMG data between subjects. The low torque level ensured that levels of co-contraction were also low relative to a normal MVC and in the same order of magnitude as those used during subsequent experimental trials.

\section{Procedure}

Subjects were positioned in the manipulandum, as described above, and instructed to relax their limb and not to assist or retard the limb perturbations. Two minutes of resting EMG was recorded before the perturbations. (A resting muscle was defined here by the absence of a voluntary contraction. No attempt was made to eliminate EMG using visual feedback.) Two consecutive 60 second perturbations of the limb were then recorded while the muscles were again at rest. In subsequent trials, subjects were instructed to maintain a constant target level of background contraction of the elbow flexor muscles against the resistance of the manipulandum throughout each perturbation of 60 seconds. A digital display of the root mean square and integrated biceps brachii muscle activity was provided to the subjects, and they were required to match this display to the target contraction level. Target contractions were set at approximately $2.5 \%, 5 \%, 7.5 \%, 10 \%$, $12.5 \%$, and $15 \%$ of MVC in normal subjects. Because of weakness in the stroke subjects, this meant that the target contraction levels frequently exceeded $15 \%$ of their MVC. In addition, because of persisting background EMG activity while attempting to rest, spastic subjects were frequently unable to achieve the lowest of these targets, resulting in fewer trials from these subjects. However, the accuracy of matching target contraction levels was not crucial to the results, and the true mean rectified EMG level for each perturbation was determined, in $\mu \mathrm{V}$ and equivalent torque units, by software during data analysis.

One minute of rest was allowed after four trials or as requested by the subject. On average, eight trials were performed.

\section{Data analysis}

The raw EMG signals were processed to produce a smoothed envelope of the raw signal, proportional to the level of muscle contraction (high pass filtered at $60 \mathrm{~Hz}$, detrended, full wave rectified, and then low pass filtered at $3 \mathrm{~Hz}$, using fourth order Butterworth filters). The torque and position data were passed through the same low pass filter before all signals were resampled at a 10th of the original sampling rate. Mean flexor and extensor EMG levels were digitally calculated at each contraction level.

Figure 1 shows samples of raw data. Cross correlation analysis $^{32}$ was performed to analyse the relations between the stretch stimulus and the flexor EMG, extensor EMG, and torque signals. The analysis provided measures of coherence square, gain, and phase between the signals. The output of the analysis for the stretch perturbation and torque signals is shown in fig $2 \mathrm{~A}-\mathrm{C}$, with the power spectrum of the perturbation signal shown in fig 2D. The estimates are plotted as a function of stretch frequency. The coherence between the signals at each frequency was defined as the proportion of signal variance accounted for by the linear relation to the stretch stimulus (fig $2 \mathrm{~A}$ ). Gain was the ratio of the amplitude of the response to the amplitude of the stretch signal at each frequency (fig $2 \mathrm{~B}$ ). Phase estimated in degrees the advance or lag of the response with respect to the stretch for each frequency (fig 2C). Individual gain and phase estimates at any frequency with a coherence of less than 0.2 were eliminated from the analysis because of their low reliability as assessed from their 95\% confidence intervals.

The viscoelastic resistance of the elbow joint was measured in two ways: first, via the torque angle gain (the ratio of the resistive torque and stretch signals) and, secondly, via the resonant frequency. The viscoelastic resistance estimates were also normalised for limb volume, estimated from volume measurements of each limb. All torque data presented in the results were normalised in this manner.

Joint resistance was also estimated by calculation of resonant frequency. At the resonant frequency, the resistive torque is a minimum because of the cancellation of elastic and inertial torques. Thus, the resonant frequency was determined as the frequency corresponding to a torque angle phase difference of $90^{\circ} .{ }^{33}$ The resonant frequency increases with elastic tissue resistance and has thus been used as a measure of stiffness. ${ }^{34}$ It has the advantage of being a simple method to isolate the elastic stiffness component from frictional and inertial components of limb and measuring equipment. 

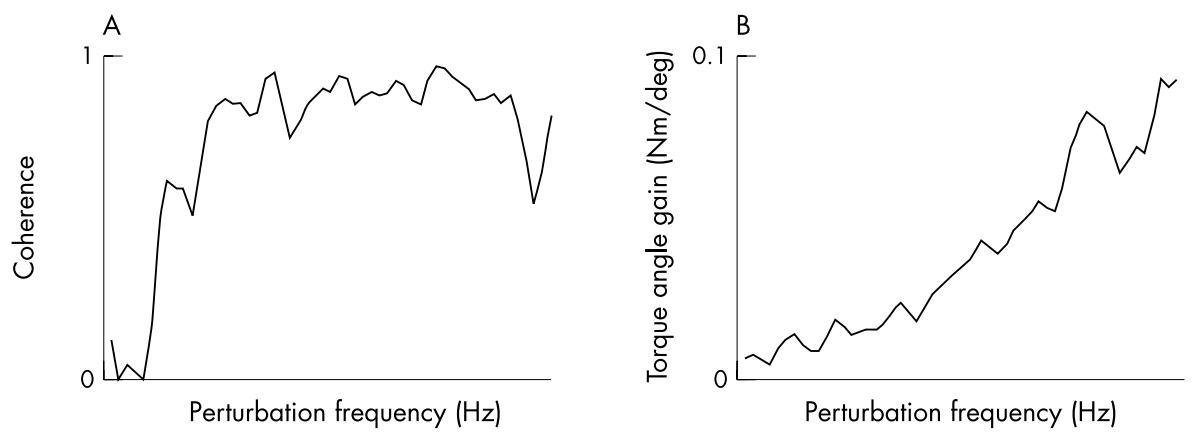

Figure 2 Estimates from correlation analysis of resistive torque and joint angle plotted against the frequency of the perturbation. (A) Coherence square, (B) torque angle gain, (C) phase difference, (D) spectral content of the position signal.
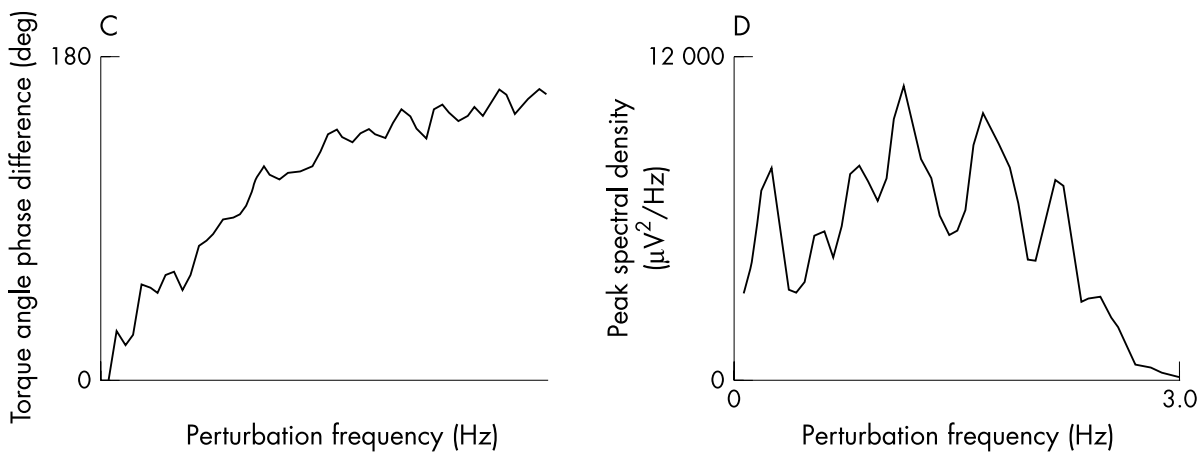

\section{Statistical analysis}

Descriptive and statistical analyses of the results were carried out using linear regression and ANOVA.

\section{RESULTS}

All EMG measurements were normalised using the torque based calibration procedure described in the methods. This showed that the normal and stroke groups generated similar levels of biceps and triceps muscle activity in $\mu \mathrm{V} / \mathrm{Nm}$ of torque (table 2). Thus, there was no significant change in the EMG-torque relation in the stroke group. The MVCs of the biceps and triceps muscles were converted to torque units using the individual biceps and triceps EMG/torque gains. It was estimated using this method that the stroke group could produce approximately $20 \%$ of the normal maximal flexor and extensor torques. This was consistent with estimates of weakness derived from the MVCs for biceps and triceps measured in $\mu \mathrm{V}$ units. Stroke subjects produced on average only $25 \%$ of the normal MVC in biceps and triceps (table 2 ).

Because the EMG/torque conversion factor varied little between individuals, the statistical results were the same for both $\mu \mathrm{V}$ and torque units. Thus, the EMG data in the following sections are presented for simplicity in $\mu \mathrm{V}$ rather than torque units.

To determine the sensitivity of the experimental methods and analysis, the effect of the smallest voluntary contraction $(2.5 \% \mathrm{MVC})$ on reflex gain and joint resistance was

Table 2 Resting unperturbed data

\begin{tabular}{lrrl}
\hline & N & S & p Value \\
\hline Biceps torque calibration $(\mu \mathrm{V} / \mathrm{Nm})$ & 17 & 15 & 0.93 \\
Triceps torque calibration $(\mu \mathrm{V} / \mathrm{Nm})$ & 20 & 16 & 0.28 \\
Biceps $\mathrm{MVC}(\mu \mathrm{V})$ & 137 & 34 & 0.00001 \\
Triceps $\mathrm{MVC}(\mu \mathrm{V})$ & 112 & 28 & 0.00001 \\
Mean resting biceps activity $(\mu \mathrm{V})$ & 0.6 & 1.9 & 0.01 \\
Mean resting triceps activity $(\mu \mathrm{V})$ & 0.9 & 1.9 & 0.35 \\
\hline
\end{tabular}

$p$ Values were calculated using one way ANOVA.

MVC, maximum voluntary contraction; $N$, normal limbs; $S$, spastic limbs following stroke. investigated in normal subjects. Normal group mean biceps gain increased from $0.05 \mu \mathrm{V} / \mathrm{deg}(\mathrm{SD}, 0.04$ ) at rest to $0.28 \mu \mathrm{V} /$ $\operatorname{deg}(\mathrm{SD}, 0.23)$ at $2.5 \%$ MVC ( $<<0.001$; one way ANOVA) and resistive torque increased from $0.06 \mathrm{Nm} / \mathrm{deg}$ (SD, 0.02) at rest to $0.09 \mathrm{Nm} / \mathrm{deg}(\mathrm{SD}, 0.06)$ at $2.5 \% \mathrm{MVC}(\mathrm{p}<0.05$; one way ANOVA). Hence, both reflex gain and joint resistance were significantly increased above resting values by a contraction as small as $2.5 \%$ MVC. This shows that the $2.5 \%$ MVC increments between target contractions were sufficient to produce significant effects on both the stretch reflex and joint mechanics.

\section{Reflex amplitude}

Flexor and extensor TSR gain showed a non-linear increase with perturbation frequencies across the range $0-3 \mathrm{~Hz}$. This gain-frequency relation (fig 3A) was fitted using least squares by a first order polynomial and the coefficients and intercepts then plotted against contraction level in each subject. The two coefficients and the intercept were linearly related to contraction level in normal and spastic muscles $(\mathrm{p}<0.0001$; linear regression $)$. The slopes were not significantly different in normal and spastic muscles.

A simpler analysis of reflex amplitude was to average the TSR gain over all frequencies and to plot the mean gains against the mean biceps background activity. The analyses of these data produced the same conclusions as the method described above. Because the units $(\mu \mathrm{V} / \mathrm{deg})$ are more intuitive and easily related to past studies, they will be used below. Regression analysis confirmed the linearity of the relation between biceps TSR gain and contraction level in individual subjects. Individual $r^{2}$ values averaged 0.89 for the normal group and 0.81 for the stroke group. The slopes of individual regression lines were variable within normal and stroke groups. When compared by one way ANOVA, the normal and stroke groups were found to have similar slopes $(\mathrm{p}=0.86)$ and similar $\mathrm{Y}$ intercepts $(\mathrm{p}=0.24)$ (representing zero contraction level).

The group data relating biceps reflex gain and contraction level were obtained for the spastic and normal groups by pooling their individual regression statistics. Figure 4 illustrates the main findings. When plotted on the same axes 

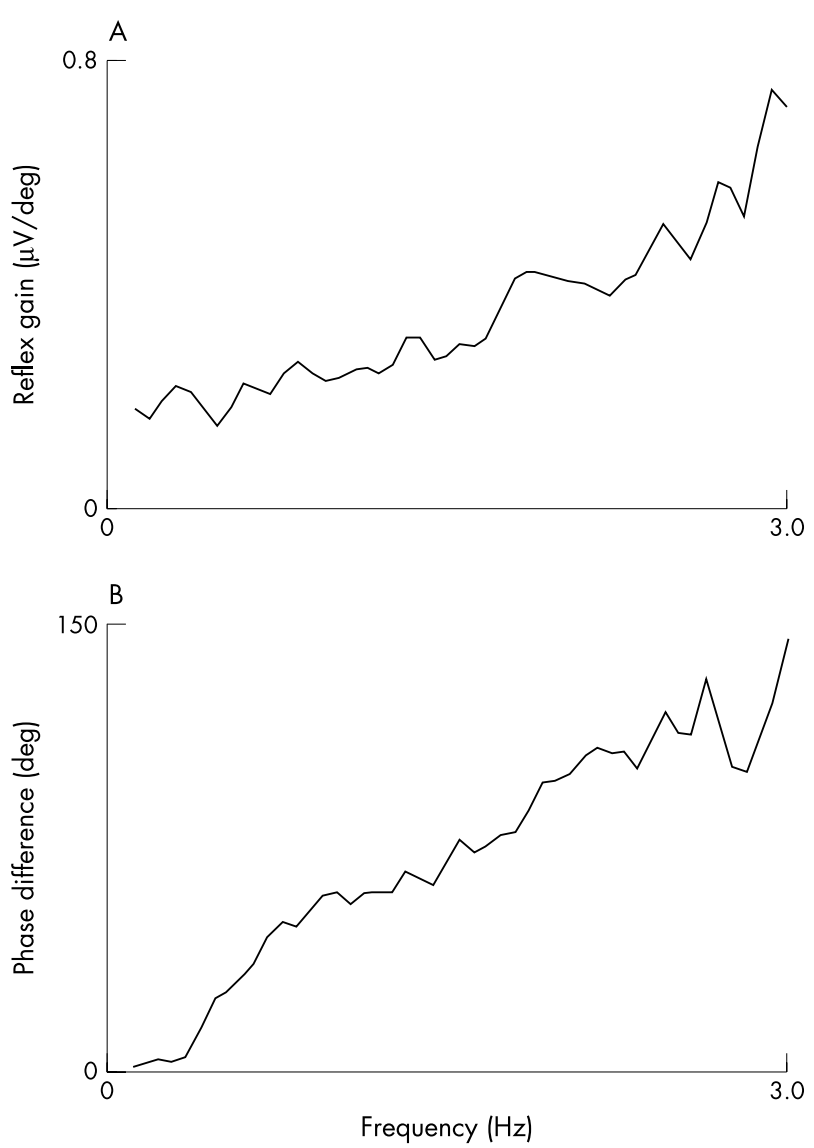

Figure 3 (A) Tonic stretch reflex (TSR) gain plotted against the frequency of the perturbation in a typical normal subject during a small $2.5 \%$ maximum voluntary contraction background contraction. (B) Phase difference of the TSR with respect to joint angle (stretch). A typical result is shown, increasing phase advance of the reflex with increase in frequency.

(fig 4A), their similar regression slopes showed that the gain was similar in both groups for any equivalent contraction level. However, it was clear that the lower limit was raised in the spastic group because of an increase of background contraction levels at rest, and the upper limit was decreased because of their lower MVC secondary to weakness. In the normals, the lower limit of gain modulation was not significantly different to zero and the upper limit was taken to be $50 \%$ of the group mean MVC. This estimate is consistent with a recent report on maximum reflex gain in a group of normal subjects. ${ }^{21}$ The figure illustrates that the available range of biceps reflex gain modulation was reduced in the spastic group in proportion to their reduced range of contraction.

During the perturbation trials, the level of triceps muscle contraction was always less than $25 \%$ of the active biceps level, but still tended to increase with the biceps contraction level in the normal and stroke groups. As with biceps, regression analysis indicated a highly linear relation between TSR gain in triceps and the triceps contraction level. A one way ANOVA of individual subjects' slopes and intercepts again showed no significant difference in slope $(p=0.85)$ or Y intercept $(p=0.22)$ between the groups. That is, triceps reflex gain was similar in both groups over the range of contractions studied.

\section{Reflex timing}

During active contraction, the TSR phase was advanced with respect to muscle stretch. That is, the peak reflex EMG burst preceded the peak of muscle stretch (fig 3). When averaged over all frequencies, the mean phase advance was approximately $100^{\circ}$ in both groups and did not change significantly with the level of biceps contraction.

The most impressive difference between the groups was in the resting data. In the resting normals, the reflex phase was retarded by approximately $100^{\circ}$ relative to muscle stretch. However, in the resting stroke subjects, the reflex phase more closely resembled that seen in normals during contraction. They did not show the abrupt change from phase lag to phase lead seen in the normals with the onset of contraction ( $\mathrm{p}<0.001$; one way ANOVA).

A similar pattern was seen in the phase of the triceps. Again, there was no significant difference between groups. Stroke subjects demonstrated a phase lead at rest, similar to the extensor phase in normal subjects during small voluntary contractions. This difference between groups in flexor and extensor EMG phase angle was significant $(p<0.001$; one way ANOVA).

\section{Mechanical resistance}

The torque angle gain of the elbow joint depended on the frequency of the perturbation. At low frequencies, the torque angle gain was at a minimum and the torque was approximately in phase with position. Torque angle gain increased with frequency and the torque angle phase shifted toward $180^{\circ}$ (fig 2B, C). These effects of frequency are predicted by the dominant contribution of tissue elasticity to resistive torque at low frequencies, and an increasing inertial contribution at higher frequencies according to the relation I $\alpha \mathrm{f}^{2}$ (where $\mathrm{I}$ is inertial resistance and $\mathrm{f}$ is the perturbation frequency). ${ }^{33}$ An increase in tissue resistance would be reflected by an increased torque angle gain in the low frequency range and a shift in the inertial phase transition towards higher frequencies. Therefore, torque angle gain was averaged across the frequency range $0.1-0.3 \mathrm{~Hz}$ (where gain was relatively independent of frequency and the inertial contribution was approximately zero) to estimate elastic resistance.

The linearity of the relation between elastic resistance and biceps contraction level was also confirmed by regression analysis in individual subjects. The mean slope of the individual regression lines was lower in the stroke group than in normals (indicating reduced elastic resistance), but the difference was not significant $(p=0.58$; one way ANOVA). Similar positive Y intercepts were obtained in both groups, indicating similar joint resistance at zero contraction level ( $\mathrm{p}=0.94$; one way ANOVA).

This conclusion was further supported by the findings for resonant frequency, calculated under resting and contracting conditions. When plotted against contraction level for each subject, the resonant frequency also showed a linear increase with increasing contraction level, reflecting an increase in active tissue resistance. Individual regression analysis was used to compare the relation between resonant frequency and contraction level in the two groups. The slopes of the regression lines were not significantly different $(p=0.69$; one way ANOVA). The positive $\mathrm{Y}$ intercepts were also very similar between groups ( $\mathrm{p}=0.97$; one way ANOVA).

The mean data relating resonant frequency and biceps contraction level were obtained for the spastic and normal groups by pooling their individual data. When plotted on the same axes (fig 4B), their similar regression slopes showed that the resonant frequency was similar in both groups for any equivalent contraction level. However, it was clear that the available range of joint stiffness modulation was reduced in the spastic group. Hence, joint stiffness would be significantly different only below or above the reduced range 

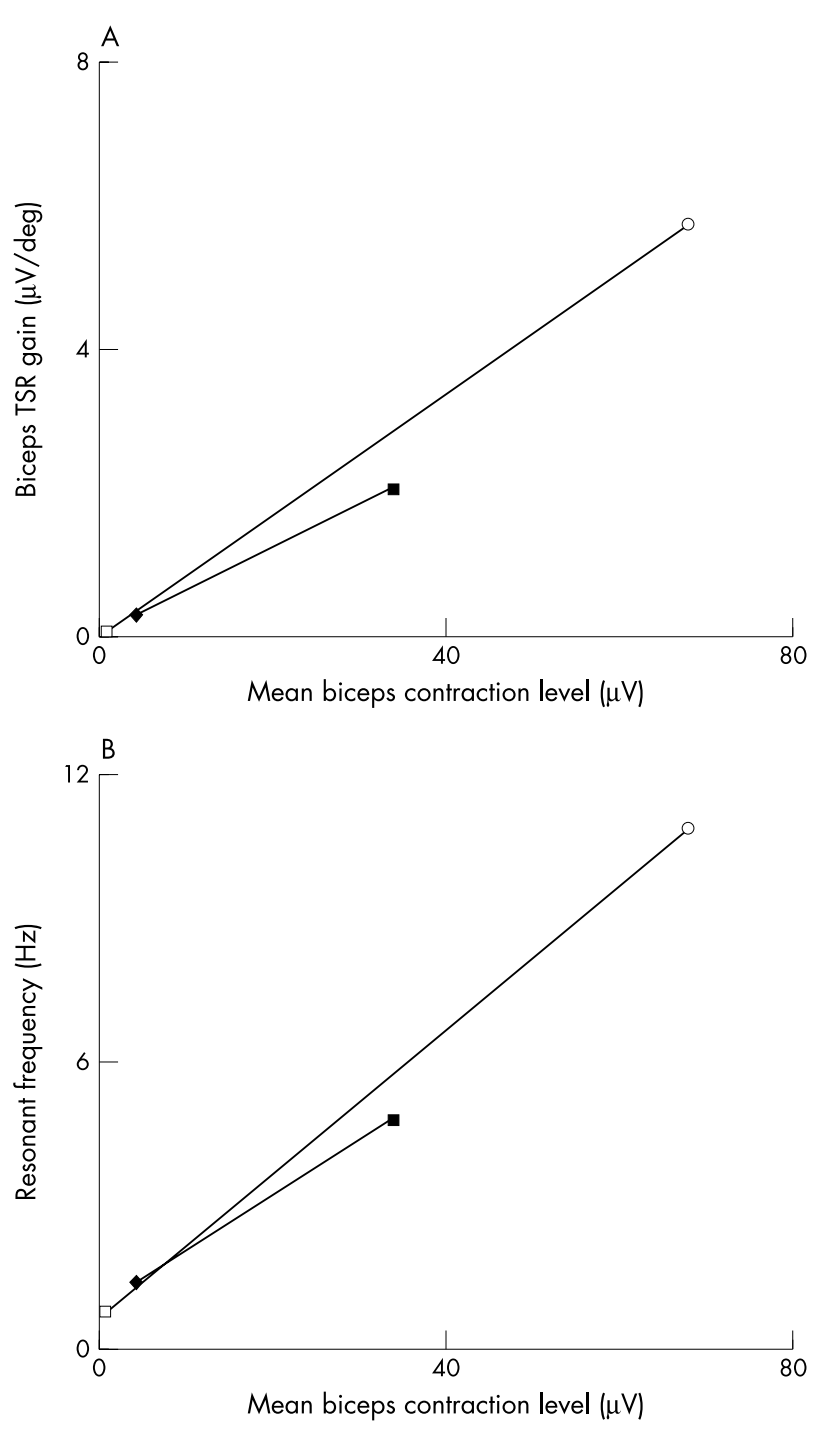

Figure 4 Linear regression lines from the pooled data relating (A) biceps tonic stretch reflex (TSR) gain and (B) joint stiffness (resonant frequency) to mean biceps contraction level in normal (open circle) and spastic (closed square) limbs. In both graphs, the slopes and zero intercepts are not significantly different, but the resting values are significantly different (see text). The figure illustrates that group differences in TSR gain or passive stiffness may be expected only above or below the reduced available range of background muscle contraction in stroke.

of contraction available to the spastic group. The first situation will be the case at "rest".

\section{Resting perturbation data}

To verify that the members of the experimental group were in fact spastic, as defined clinically, the data from the resting condition were compared in spastic and normal groups. This confirmed the findings of many past studies. The mean resting biceps TSR gain and background EMG levels were significantly increased, both approximately fivefold, in the stroke group during the perturbation $(\mathrm{p}<0.0001$; one way ANOVAs). The resting mechanical joint resistance (torque angle gain) was also significantly increased, approximately twofold ( $p<0.0001$; one way ANOVA). Furthermore, a significant positive relation was found between the resting torque angle gain and the resting biceps $(p<0.0001$; linear regression) and triceps $(\mathrm{p}<0.001$; linear regression) $\mathrm{EMG}$ levels. Therefore, it can be concluded that the raised background contraction levels in the stroke group were related to both increased reflex gain and joint resistance.

\section{Resting pre-perturbation data}

Given the clear association between traditional measures of spasticity and raised background contraction levels during the perturbation, the mean resting levels of background contraction were analysed in the biceps and triceps muscles over the two minutes before the perturbation. In both muscles, the mean contraction level was higher in stroke subjects than in normal subjects, the difference being significant in biceps but not triceps (table 2 ).

Raised background contraction levels in resting stroke subjects during the perturbation were significantly related to the raised resting contraction levels in the same muscles before the perturbation and inversely related to the MVC in biceps $(p<0.05$; linear regression $)$. Hence, in stroke subjects, a small MVC and weakness were related to raised resting contraction levels and reflex gain in the same muscle.

\section{DISCUSSION}

The major difference found between the normal and spastic group, other than significant weakness in the last group, was a modest rise in the background levels of contraction when spastic limbs were at rest. As a consequence of this raised background contraction, when perturbations with amplitudes and frequencies comparable to those used clinically were imposed on the resting limb, the stretch reflex and the passive joint resistance were increased in the spastic subjects. However, the magnitudes of these increases were entirely in agreement with those predicted by regression analysis from the modest rise in background contraction. When the background contraction level was comparable, both reflex gain and joint mechanical resistance were also comparable between the two groups, so that there was no evidence of an increase in the intrinsic stretch reflex loop gain or passive joint resistance in the spastic group.

The results confirm earlier studies that found an approximately linear relation between reflex amplitude and low levels of voluntary contraction in normal subjects. ${ }^{16} 2123-29$ Furthermore, they show that this relation holds also in stroke. The finding that the slopes of the regression lines relating reflex gain and joint resistance to contraction level were not significantly different in the normal and stroke groups over the tested range means that both reflex amplitude and joint resistance fell within the normal range for any contraction level achieved by spastic subjects. This provides strong evidence that the intrinsic loop gain of the stretch reflex is not increased in spastic subjects.

The findings of our study underline the need to control for differences in contraction levels when comparing reflexes in different groups. There have been no attempts previously to quantify "resting" EMG levels or to assess the impact of incomplete relaxation on reflex gain or passive tissue resistance estimates in stroke subjects, although persisting activity in resting spastic muscles has been reported. ${ }^{35}$ The group mean biceps background contraction during perturbations applied when spastic subjects attempted to relax was equivalent to $3.1 \%$ of the normal MVC (in the normal subjects it was $0.6 \%$ ). Therefore, the spastic subjects exceeded the level required to increase reflex gain and joint resistance significantly in normals, where a voluntary background contraction of $2.5 \%$ of MVC produced significant increases in biceps reflex gain and joint resistance. The resting biceps reflex gain in spastic subjects was approximately five times higher than normal resting values. This increase was sufficient to influence joint mechanics, because resting reflex gain significantly correlated with resting joint resistance levels in the spastic limbs, but not the normal limbs. It can be 
concluded that the rise in resting background muscle activity is a primary factor in the greater prominence of spastic signs (viz, increased reflex gain and passive joint resistance) in resting rather than in contracting muscle, as seen here and in earlier studies.

Linear regression analysis showed that the maximal achievable reflex gain in spastic subjects was decreased proportionately to the degree of weakness. Their mean MVC was approximately $25 \%$ of the normal value in our study. Thus, if reflex gain is compared with normal muscles at high contraction levels that exceed the limited range of gain modulation in stroke, a relative decrease in gain may be observed. $^{2}{ }^{17} 18$ Similarly, because joint resistance tended to increase in a linear manner with contraction level, there will be a reduction in the available range of joint stiffness modulation, with significant functional implications.

Also evident from our study, is the importance of the method used to calibrate the EMG when comparing normal and stroke subjects. Differences in calibration methods must have contributed to the conflicting findings on spasticity in previous studies. Calibration against MVC would have greatly altered the interpretation of the data reported in our present study. Such a method is of course invalidated by significant weakness in the stroke group. In view of the finding that the relation between torque production and EMG voltage was not significantly altered by weakness, EMG voltage was an appropriate unit for comparison.

Under the experimental conditions, there was no evidence that co-contraction of antagonist muscles or increased reflex activity in antagonist muscles contributed to spasticity. The ratio of mean flexor to extensor activity was similar in stroke and normal subjects at all levels of flexor contraction. Regression analysis in both flexor and extensor muscles of normal and stroke subjects showed that reflex gain was linearly related to contraction level, and that the slopes of these regressions were not significantly different in flexor and extensor muscles. The TSR phase analysis also gave no indication of an abnormal reciprocal relation between antagonist muscles. A shift in their relative phase would have provided evidence of abnormal reciprocal mechanisms, but both muscles maintained a normal phase advance of approximately $100^{\circ}$ when contracted..$^{21}$ Therefore, our findings suggest that co-contraction does not occur as a result of changes in segmental reciprocal reflex mechanisms. This suggests that the abnormal patterns of co-contraction of muscle antagonists previously reported in spasticity ${ }^{36-38}$ may be task specific, and thus of supraspinal origin, because they are not a feature of the simple isometric contractions studied here.

In our study, it was possible to estimate reflex gain, torque angle gain, and resonant frequency at a theoretical zero contraction level, by extrapolating their respective regression lines with respect to contraction level. No significant differences between groups were found. The intercept on the torque angle gain axis reflects the passive joint resistance component, and the findings indicate that it was small relative to active resistance in the resting stroke group. A small increase in passive resistance is unlikely to be detected by relatively insensitive clinical scales, and therefore is unlikely to contribute significantly to the clinical picture of hypertonia. Given et al estimated passive resistance at the elbow and also found no difference between normal and stroke subjects. ${ }^{39}$ Although several studies have reported significant passive tissue contributions to spasticity, ${ }^{39-45}$ the degree of relaxation in the subjects was not measured, except by visual inspection of raw EMG records. As the results of our present study show, without accurate matching of contraction levels, normal and spastic groups cannot be compared reliably.

However, an important measurement with regard to the question of passive resistance is the selection of subjects. Our current study is based on limbs that were free of significant contracture, as assessed clinically, and were able to be moved repeatedly through a range of $30^{\circ}$ without significant discomfort. Therefore, the results do not bear on the potential contribution of passive tissue changes to spasticity in limbs with substantial contracture. The degree of contracture is probably an important variable in determining the relative magnitude of the passive component.

An increase in the velocity sensitivity of the stretch reflex has been reported in spasticity. ${ }^{4}$ This has been attributed to an increase in the slope of the TSR-velocity relation with increasing severity of spasticity. ${ }^{19}{ }^{46}$ No evidence of a difference in the slope of the gain-frequency relation between normal and spastic muscle was found in our current study. TSR gain increased in a non-linear manner with perturbation frequency in normal (see also Neilson and Lance and Neilson and McCaughley ${ }^{1627}$ ) and spastic muscles, but the slopes of the gain-frequency regression lines and their intercepts were not significantly different at equivalent contraction levels. Because the contraction levels in spastic muscle in the resting condition were not equivalent to normal, the effect was to displace the non-linear reflex gain-frequency relation upward on the $\mathrm{Y}$ axis and increase its slope. Thus, reported increases in velocity sensitivity and also of reduced velocity threshold ${ }^{18}{ }^{46-48}$ in spastic muscles may be explicable in terms of a normal velocity dependence of the TSR in the presence of a raised resting contraction level.

The rationale for the use of antispastic agents in treatment is that upper or lower limb performance is compromised by excessive spastic muscle activity of involuntary or reflex origin, and that performance may be improved by reducing this activity. Implicit in this approach is the view that the exaggerated reflex activity apparent at rest will translate into exaggerated reflex activity during voluntary movements. Some previous studies ${ }^{2}{ }^{17-19}$ have failed to demonstrate such increased activity under active conditions, and our present study supports and extends these findings. With accurate matching of background contraction between subjects, so as to assess the peripheral manifestations of spasticity independently of descending supraspinal influences, we found no evidence of increased muscle activity as reflected by increased mechanical resistance or increased stretch reflex loop gain. Therefore, the rationale for the routine use of antispastic agents was not supported.

It is clear that dynamic modulation of muscle reflexes occurs during movement tasks and that deficits in dynamic reflex modulation may accompany spasticity. ${ }^{49}$ We have shown that the range of reflex gain modulation is reduced in spasticity as a result of raised resting contraction levels and weakness. Hence, task performance in the spastic movement disorder is more likely to be limited by disturbed central modulation of reflex activity than by a persisting increase in reflex activity (fig 4A, B).

An important qualification of the results is that the subjects selected for our study did not have significant degrees of contracture, so that the results do not exclude the possible contribution of muscle shortening and reduced range of movement to disability in some patients.

\section{ACKNOWLEDGEMENTS}

The authors would like to thank all subjects who gave of their time to assist in this study. The authors are also grateful to Dr W Zev Rymer for helpful comments on the manuscript.

\section{Authors' affiliations}

J A Burne, V L Carleton, School of Biomedical Sciences, University of Sydney, PO Box 170, Lidcombe, NSW 1825, Australia 
N J O'Dwyer, School of Exercise and Sport Science, University of Sydney

Competing interests: none declared

\section{REFERENCES}

1 Lance JW. Symposium synopsis. In: Feldman RG, Young RR, Koella WP, eds. Spasticity: disordered motor control. Miami: Symposia Specialists, 1980:485-94.

2 Ada L, Vattanasilp W, O'Dwyer N, et al. Does spasticity contribute to walking dysfunction after stroke? J Neurol Neurosurg Psychiatry 1998;64:638-5.

3 Apkarian JA, Naumann S. Stretch reflex inhibition using electrical stimulation in normal subjects and subjects with spasticity. J Biomed Eng 1991;13:67-73.

4 Ibrahim IK, Berger W, Trippel M, et al. Stretch-induced electromyographic activity and torque in spastic elbow muscles. Brain 1993;1 16:971-89.

5 Meyer M, Adorjani C. Tonic stretch reflex for quantification of pathological muscle tone. In: Feldman RG, Young RR, Koella WP, eds. Spasticity: disordered motor control. Miami: Symposia Specialists, 1980:315-30.

6 Meyer M, Adoriani C. Quantification of the effects of muscle relaxant drugs in man by tonic stretch reflex. In: Desmedt JE, ed. Motor control mechanisms in health and disease. Advances in neurology. New York: Raven, 1983:9.

7 Neilson PD. Interaction between voluntary contraction and tonic stretch reflex transmission in normal and spastic patients. J Neurol Neurosurg Psychiatry 1972;35:853-60.

8 Neilson PD. Voluntary and reflex control of the biceps brachii muscle in spastic-athetotic patients. J Neurol Neurosurg Psychiatry 1972:35:589-98.

9 Neilson PD. Tonic stretch reflex in normal subjects and in cerebral palsy. In Gandevia SC, Burke D, Anthony M, eds. Science and practice in clinical neurology. London: Cambridge University Press, 1993:169-90.

10 Brown TIH, Rack PMH, Ross HF. Electromyographic responses to imposed sinusoidal movement of the human thumb. Physiotherapy 1982;332:87-99.

11 Burke D. Spasticity as an adaptation to pyramidal tract injury. In: Waxman SG, ed. Functional recovery in neurological disease. Advances in neurology. New York: Raven Press, 1988:401-22.

12 Clemmesen $\mathbf{S}$. Some studies on muscle tone. Proc $R$ Soc Med 1951;44:637-46.

13 Ralston HJ, Libet $\mathrm{H}$. The question of tonus in skeletal muscle. Am J Physical Med 1953;32:85-92

14 Yeo W, Ada L, O'Dwyer N, et al. Tonic stretch reflexes in older able-bodied people. Electromyogr Clin Neurophysiol 1998;38:273-8.

15 Gottlieb GL, Agarwal GC. Dependence of human ankle compliance on joint angle. J Biomechanics 1978;11:177-81

16 Neilson PD, Lance JW. Reflex transmission characteristics during voluntary activity in normal man and patients with movement disorders. In: Desmedt JE ed. Cerebral motor control in man: long loop mechanisms. Progress in clinical neurophysiology. Basel: Karger, 1978:263-99.

17 Dietz V, Trippel M, Berger W. Reflex activity and muscle tone during elbow movements in patients with spastic paresis. Ann Neurol 1991;30:767-78.

18 Lee WA, Boughton A, Rymer WZ. Absence of stretch reflex gain enhancement in voluntarily activated spastic muscle. Exp Neurol 1987;98:317-35.

19 Powers RK, Campbell DL, Rymer WZ. Stretch reflex dynamics in spastic elbow flexor muscles. Ann Neurol 1989;25:32-42.

20 Sinkjaer T, Toft E, Larsen K, et al. Non-reflex and reflex mediated ankle joint stiffness in multiple sclerosis patients with spasticity. Muscle Nerve 1993;16:69-76.

21 Cathers I, O'Dwyer N, Neilson P. Variation of magnitude and timing of wrist flexor stretch reflex across the full range of voluntary activation. Exp Brain Res 2004 [In press].

22 Kearney RE, Chan CWY. Contrasts between the reflex responses of tibialis anterior and triceps surae to sudden ankle rotation in normal human subjects. Electromyogr Clin Neurophysiol 1982;54:301-10.

23 Kearney RE, Hunter IW. System identification of triceps surae stretch reflex dynamics Exp Brain Res 1983:51:117-27.

24 Marsden CD, Merton PA, Morton HB. Servo action in human voluntary movement. Nature 1972;238:140-3.

25 Marsden CD, Merton PA, Morton HB. Servo action in the human thumb. J Physiol (Lond) 1976;257:1-44.
26 Matthews PBC. Observations on the automatic compensation of reflex gain on varying the pre-existing level of motor discharge in man. J Physiol (Lond) 1986;374:73-90

27 Neilson PD, McCaughey J. Effect of contraction level and magnitude of stretch on tonic stretch reflex transmission characteristics. J Neurol Neurosurg Psychiatry 1981;44:1007-12.

28 Toft E, Sinkjær T, Andreassen S. Mechanical and electromyographic responses to stretch of the human anterior muscle at different levels of contraction. Exp Brain Res 1989;74:213-19.

29 Zhang L, Rymer WZ. Simultaneous and nonlinear identification of mechanical and reflex properties of human elbow joint muscles. IEEE Trans Biomed Eng 1997:44:1192-209.

30 Cathers I, O'Dwyer N, Neilson P. Dependence of stretch reflexes on amplitude and bandwidth of stretch in human wrist muscle. Exp Brain Res 1999;129:278-87.

31 Kearney RE, Hunter IW. Dynamics of human ankle stiffness: variation with displacement amplitude. J Biomech 1982;15:753-6.

32 Bendat JS, Piersol AG. Random data: analysis and measurement procedures. New York: Wiley-Interscience, 1971.

33 Joyce GC, Rack PMH, Ross HF. The forces generated at the human elbow joint in response to imposed sinusoidal movements of the forearm. J Physiol (Lond) 1974;240:351-74.

34 Lakie M, Walsh EG, Wright GW. Resonance at the wrist demonstrated by the use of a torque motor: an instrumental analysis of muscle tone in man. Physiotherapy 1984;353:265-85.

35 Pisano F, Miscio G, Del Conte C, et al. Quantitative measures of spasticity in post-stroke patients. Clin Neurophysiol 2000;111:1015-22.

36 Fellows SJ, Kaus C, Ross HF, et al. Agonist and antagonist EMG activation during isometric torque development at the elbow in spastic hemiparesis. Electroencephalogr Clin Neurophysiol 1994;93:106-12.

37 Levin MF. Interjoint co-ordination during pointing movements is disrupted in spastic hemiparesis. Brain 1996;119:281-93.

38 McLellan DL. Co-contraction and stretch reflexes in spasticity during treatment with baclofen. J Neurol Neurosurg Psychiatry 1977;40:3-38.

39 Given JD, Dewald JP, Rymer WZ. Joint dependent passive resistance in paretic and contralateral limbs of spastic patients with hemiparetic stroke. J Neurol Neurosurg Psychiatry 1995;59:271-5.

40 Dietz V, Quintern J, Berger W. Electrophysiological studies of gait in spasticity and rigidity. Evidence that altered mechanical properties of muscle contribute to hypertonia. Brain 1981;104:431-49.

41 Goldspink G, Williams PE. Muscle fibre and connective tissue changes associated with use and disuse. In Ada A, Canning C, eds. Foundations for practice: topics in neurological physiotherapy. London: Heinemann, 1990:197-218.

42 Halar EM, Stolov WC, Venkatesh B, et al. Gastrocnemius muscle belly and tendon length in stroke patients and able-bodied persons. Arch Phys Med Rehabil 1978:59:476-84.

43 Hufschmidt A, Mauritz KH. Chronic transformation of muscle in spasticity: a peripheral contribution to increased tone. J Neurol Neurosurg Psychiatry 1985;48:676-85

44 O'Dwyer NJ, Ada L. Reflex hyperexcitability and muscle contracture in relation to spastic hypertonia. Curr Opin Neurol 1996;9:451-5.

45 O'Dwyer NJ, Ada L, Neilson PD. Spasticity and muscle contracture following stroke. Brain 1996;119:1737-49.

46 Thilmann AF, Fellows SJ, Garms E. The mechanism of spastic muscle hypertonus. Variation in reflex gain over the time course of spasticity. Brain $1991 ; 114: 233-44$.

47 Powers RK, Marder-Meyer J, Rymer WZ. Quantitative relations between hypertonia and stretch reflex threshold in spastic hemiparesis. Ann Neurol 1988;23:115-24.

48 Thilmann AF, Fellows SJ, Garms E. Pathological stretch reflexes on the "good" side of hemiparetic patients. J Neurol Neurosurg Psychiatry 1990:53:208-14.

49 Morita H, Crone C, Christenhuis D. Modulation of presynaptic inhibition and disynaptic reciprocal la inhibition during voluntary movement in spasticity. Brain 2001;124:826-37. 